Recibido:

$11-|| \mid-2019$

Aceptado:

18-III-2019

Publicado en línea:

22-1I-2019
Infiltrantes para tratamiento estético de lesiones

de manchas blancas por fluorosis: Reporte de caso

\title{
Infiltrants for Aesthetic Treatment of White Spots Lesions \\ by Fluorosis: Case Report
}

César Pomacóndor-Hernández DDS, MSc, PhD;

Natália Maria Aparecida Hernandes da Fonseca DDS, MSc, PhD²

1. Dirección de Investigación, Universidad Privada Juan Pablo II, Lima, Perú.

2. Departamento de Cariología, Brazcubas Educação. Campus Mogi das Cruzes, Brasil.

Autor para correspondencia:

Dr. César Pomacóndor-Hernández - cesar.pomacondor.hernandez@gmail.com

RESUMEN: La fluorosis dental es una anomalía en el desarrollo del esmalte debido a la excesiva ingesta de flúor durante la formación de los tejidos dentales, y se caracteriza principalmente por la hipomineralización y mayor porosidad de la estructura del esmalte. Muchas veces las manchas ocasionadas por la fluorosis dental pueden comprometer la estética del paciente y afectar su autoestima. En años recientes ha sido desarrollado una nueva generación de materiales odontológicos conocida como infiltrantes resinosos, que básicamente son resinas de baja viscosidad que infiltran y paralizan la progresión de las lesiones cariosas incipientes no cavitadas. Cuando infiltrantes resinosos han sido utilizados para detener las lesiones cariosas se ha verificado que también producen cambios en la apariencia de la mancha blanca, atenuándola o tornándola imperceptible. El presente artículo describe un reporte de caso del uso de infiltrantes resinosos para tratamiento estético de lesiones de manchas blancas ocasionadas por fluorosis.

PALABRAS CLAVE: Fluorosis dental; Odontopatías; Materiales dentales; Resinas sintéticas; Metacrilatos; Estética dental. 
ABSTRACT: Dental fluorosis is an abnormality in the development of enamel due to the excessive intake of fluoride during formation of dental tissues, mainly characterized by hypomineralization and increased porosity of the enamel structure. Oftenly the spots caused by dental fluorosis can compromise the aesthetics of the patient and affect their self-esteem. In recent years a new generation of dental materials has been developed, known as resin infiltrants, which are basically low viscosity resins that infiltrate and paralyze the progression of incipient non cavitated carious lesions. When resin infiltrants have been used to stop carious lesions it has been verified that they also produce changes in the appearance of white spots, masking or making them imperceptible. The present article describes a case report of the use of resin infiltrants for aesthetic treatment of white spot lesions caused by fluorosis.

KEYWORDS: Dental fluorosis; Tooth diseases; Dental materials; Synthetic resins; Methacrylates; Dental esthetics.

\section{INTRODUCCIÓN}

La fluorosis dental es una anomalía en el desarrollo del esmalte debido a la excesiva ingesta de flúor durante la formación de los tejidos dentales. Los dientes afectados por fluorosis se caracterizan principalmente por la hipomineralización y mayor porosidad de la estructura del esmalte. El grado de severidad de la fluorosis dental está determinado por la dosis y duración de la exposición al flúor, nivel de actividad física, peso y estado nutricional del individuo, entre otros factores. Cuando el grado es leve, las lesiones ocasionadas por la fluorosis dental se caracterizan por presentarse como áreas opacas y blanquecinas. En casos moderados y graves las lesiones pueden adquirir una tonalidad marrón y volver el esmalte frágil y susceptible a la fractura $(1,2)$.

Las manchas ocasionadas por la fluorosis dental, particularmente en los dientes anterosuperiores, comprometen la estética del paciente y pueden afectar su autoestima. El tratamiento de estas manchas para conseguir una mejora estética de la sonrisa varía según el grado de severidad de la lesión. Los dientes con manchas blancas leves y de poca profundidad pueden tratarse con aclaramiento dental y microabrasión de la superficie del esmalte, mientras que las manchas fluoróticas moderadas y severas generalmente son tratadas con procedimientos mucho más invasivos como restauraciones de resina compuesta, carillas 0 coronas $(3,4)$.

En cuanto a los casos leves de fluorosis, el aclaramiento dental es la primera opción de tratamiento ya que es una estrategia no invasiva que puede esconder y enmascarar las manchas blancas. Sin embargo, dependiendo del grado de severidad de la lesión, este procedimiento no siempre llega a conseguir resultados estéticos completamente satisfactorios. Por otro lado, la microabrasión del esmalte es un tratamiento bastante utilizado y eficaz que consigue una mejora estética debido a la remoción o desgaste de la capa superficial de la lesión de esmalte (aproximadamente hasta $360 \pm 120 \mu \mathrm{m}$ ), por lo que no se le considera como un procedimiento completamente no invasivo (5).

En años recientes ha sido desarrollada una nueva generación de materiales odontológicos conocidos como infiltrantes resinosos, que básicamente son resinas de baja viscosidad creadas para infiltrar las lesiones cariosas incipientes no cavitadas, y de esta forma paralizar la progresión 
de la lesión (6). Las lesiones cariosas incipientes se caracterizan por presentar una zona desmineralizada (llamada cuerpo de la lesión) debajo de una aparente capa superficial intacta de esmalte. Los infiltrantes resinosos están compuestos principalmente por monómero trietilenglicol dimetacrilato (TEGDMA), y utilizan el principio de capilaridad para infiltrar y penetrar a través de las microporosidades del esmalte superficial intacto y rellenar el cuerpo de la lesión cariosa incipiente $(6,7)$.

Las lesiones cariosas incipientes pueden ser clínicamente diagnosticadas como manchas blancas, pues la porosidad aumentada del cuerpo de la lesión causa la característica apariencia blanquecina de estas lesiones. Cuando infiltrantes resinosos han sido utilizados para detener el progreso de lesiones cariosas se ha verificado también que producen cambios en la apariencia de la mancha blanca, atenuándola 0 tornándola imperceptible. Esta ventaja estética de los infiltrantes resinosos ha sido verificada también para enmascarar las regiones blanquecinas que dejan los brackets tras su remoción $(3,8)$.

El presente artículo describe un reporte de caso sobre el uso de infiltrantes resinosos como tratamiento estético de lesiones de manchas blancas ocasionadas por fluorosis. El objetivo es dar a conocer una nueva alternativa mínimamente invasiva de tratamiento estético, a diferencia de otros procedimientos (como la microabrasión y las restauraciones de resina compuesta) que desgastan los tejidos duros y aumentan la probabilidad de sensibilidad dental o caries secundaria. Asimismo, se busca explicar el mecanismo de acción de los infiltrantes resinosos, describir el paso a paso de su aplicación clínica, y mostrar los resultados obtenidos al finalizar el tratamiento.

\section{REPORTE DE CASO}

Un paciente de 28 años, sexo masculino, fue atendido en la Clínica Universitaria de la Facultad de Odontología de la Universidad Brasil - Campus Guarulhos, en el Estado de São Paulo - Brasil. El motivo de la consulta fue la presencia de manchas blancas en algunos dientes de la región premolar superior, lo cual le causaba fastidio a la hora de sonreír. Luego de recabar informaciones en la anamnesis y tras la realización del examen clínico detallado, las manchas blancas fueron diagnosticadas como lesiones en esmalte consecuencia de fluorosis dental de grado leve (Figura 1). En este caso fue propuesto como tratamiento mínimamente invasivo la aplicación de infiltrante resinoso en las lesiones fluoróticas para la mejora de la estética.

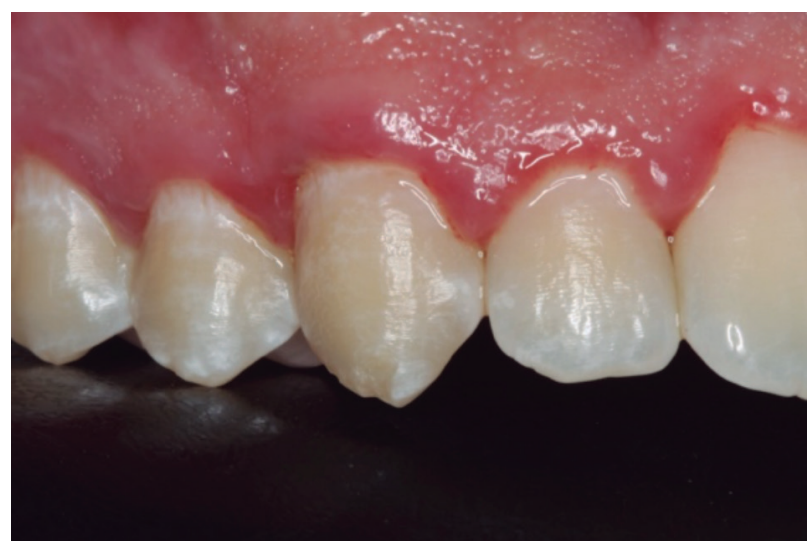

Figura 1. Manchas blancas ocasionadas por fluorosis de grado leve en las piezas dentales $15,14,13$ y 12 .

\section{APLICACIÓN DEL INFILTRANTE RESINOSO}

En el procedimiento fue utilizado el infiltrante resinoso Icon (DMG, Alemania). Este producto consta de 3 componentes (Figura 2): 1) Icon-Etch, ácido clorhídrico al 15\%; 2) Icon-Dry, etanol al 95\%; y 3) Icon-Infiltrant, infiltrante resinoso de baja viscosidad a base de TEGDMA. Tras la limpieza de Ios dientes con agua y piedra pómez, fue colocada una barrera gingival (Top Dam, FGM, Brasil) sobre las piezas dentales 12, 13, 14 y 15 (Figura 3). El tratamiento del otro cuadrante (piezas 22, 23, 24 y 25) se realizó en otra sesión y no se realizó registro fotográfico. La capa superficial de las lesiones de mancha blanca fue erosionada utilizando el ácido clorhídrico al 15\% durante 2 minutos (Figura 4). 
Luego, el ácido fue lavado usando spray de agua y aire. Tras verificar la apariencia blanquecina de aire durante 30 segundos y secado con aire libre de aceite y agua. Para contribuir con la remoción del agua remanente en las microporosidades del cuerpo de la lesión, ésta fue deshidratada con la aplicación de etanol al 95\% por 30 segundos (Figura 5) seguido por una nueva aplicación de la lesión de esmalte más pronunciada debido a la deshidratación profunda, se procedió a realizar lentamente la infiltración resinosa sobre las lesiones durante 3 minutos utilizando las puntas de aplicación especiales proporcionadas por el fabricante (Figura 6).

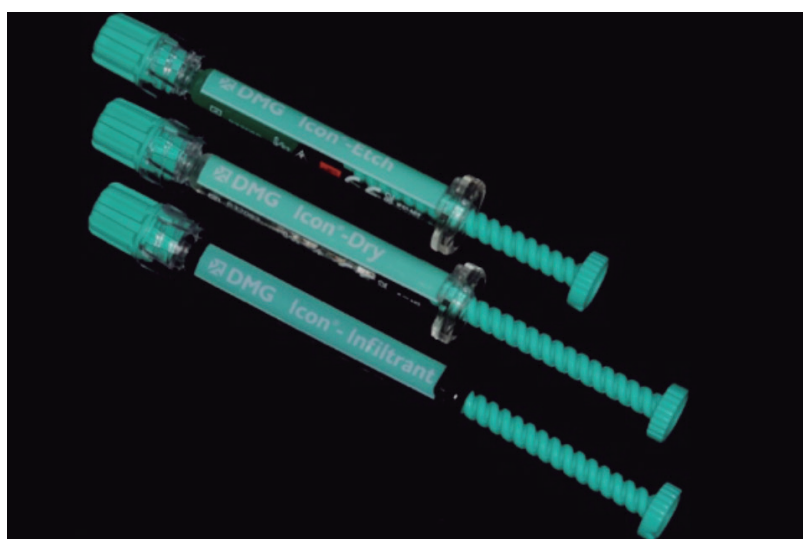

Figura 2. Infiltrante resinoso Icon (DMG, Alemania).

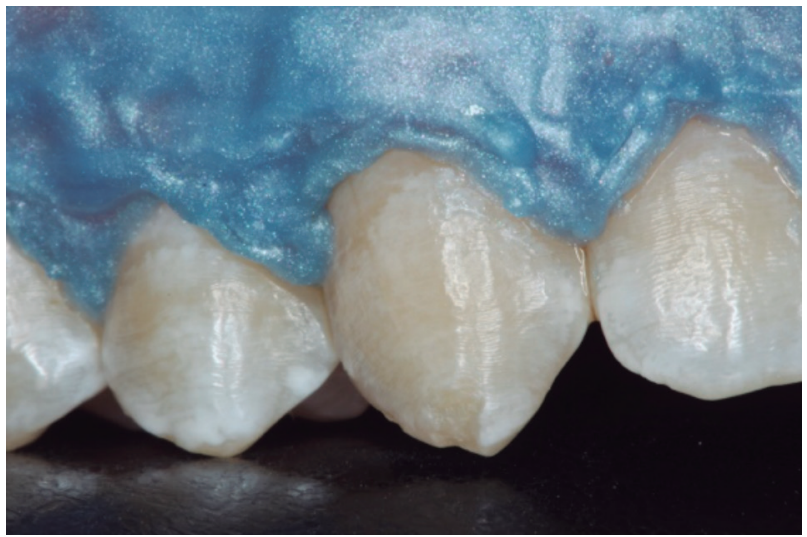

Figura 3. Barrera gingival colocada para protección del tejido blando.

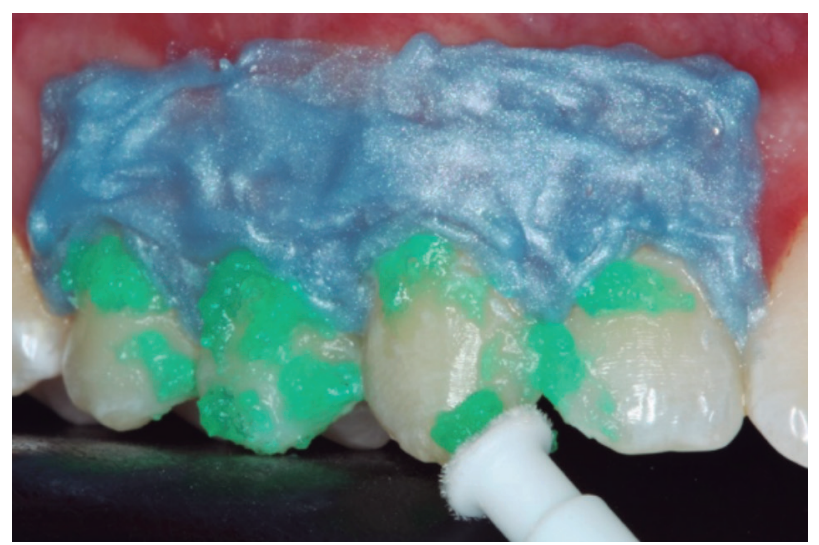

Figura 4. Aplicación del Icon-Etch (ácido clorhídrico al 15\%). 


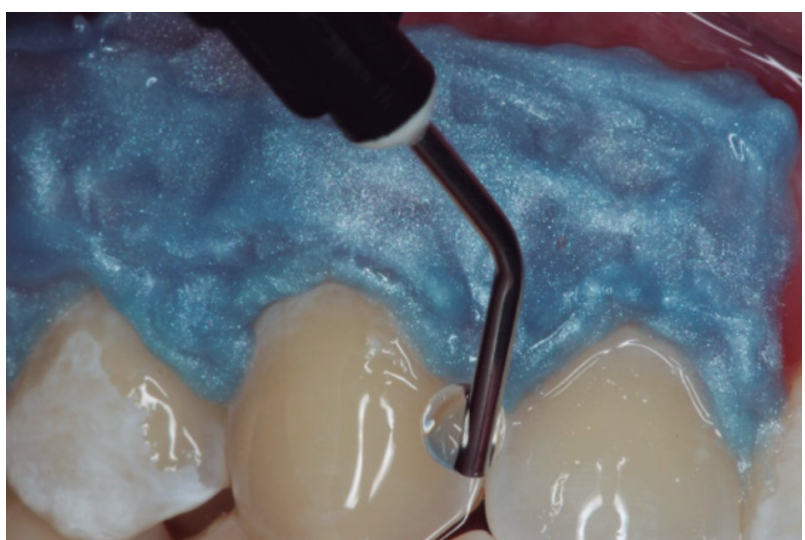

Figura 5. Aplicación del Icon-Dry (etanol al 95\%).

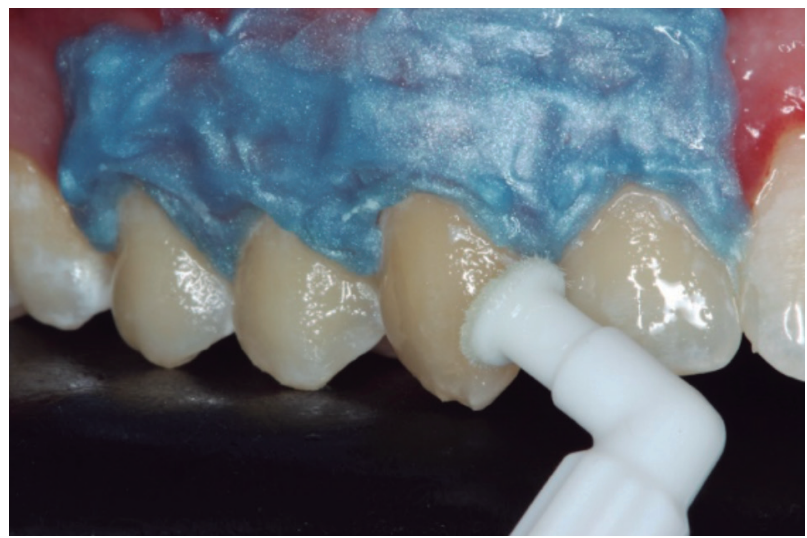

Figura 6. Aplicación del Icon-Infiltrant (infiltrante resinoso de baja viscosidad).

Los excesos groseros de infiltrante sobre las superficies dentales fueron removidos sutilmente con aire, y a continuación el infiltrante dentro de las lesiones fue fotoactivado durante 40 segundos (Figura 7). Una segunda aplicación de infiltrante resinoso fue realizada durante 1 minuto, los excesos fueron removidos y se procedió con la fotoactivación por 40 segundos. Finalmente fue realizado el pulido con puntas de silicona para eliminar zonas irregulares, la barrera gingival fue retirada y se verificó la mejora estética debido al tratamiento de infiltración resinosa (Figura 8).

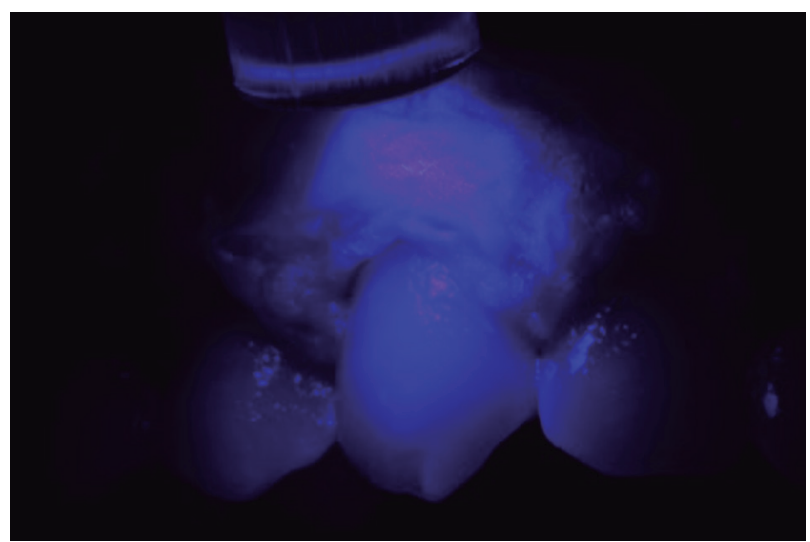

Figura 7. Fotoactivación del infiltrante resinoso en el interior de las lesiones fluoróticas.

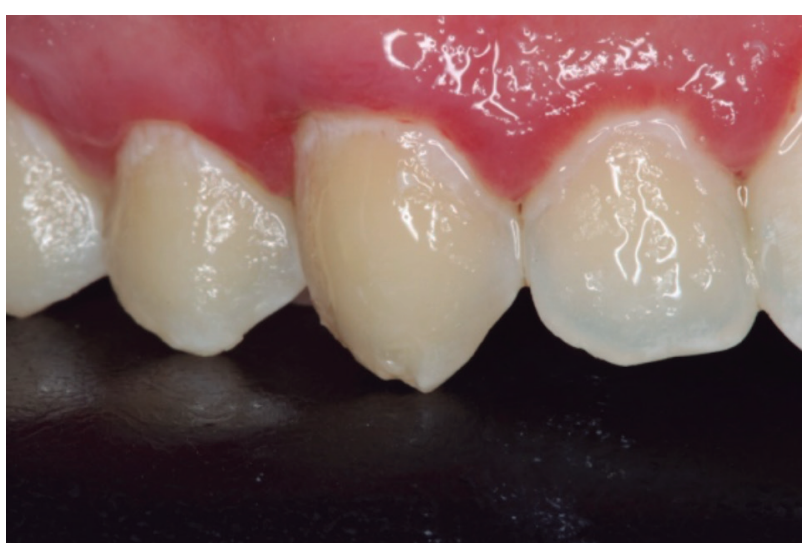

Figura 8. Caso clínico finalizado.

\section{DISCUSIÓN}

El concepto de Odontología Mínimamente Invasiva consiste en realizar tratamientos evitando el desgaste del tejido dental para garantizar la supervivencia del diente (9). Sin embargo, en la actualidad aún no se llega a un consenso sobre el tratamiento estético ideal de algunas lesiones de manchas blancas (como lesiones cariosas incipientes paralizadas, lesiones postortodóncicas, manchas causadas por fluorosis, o lesiones hipoplásicas), en las cuales muchas 
veces el tratamiento escogido es bastante invasivo mediante el desgaste del tejido alterado y su posterior restauración (1).

El aclaramiento dental es una estrategia no invasiva para esconder y enmascarar las manchas blancas, actuando a través del aclaramiento del esmalte sano, haciendo que las lesiones sean menos perceptibles. Desafortunadamente, el aclaramiento dental no siempre llega a conseguir resultados estéticos completamente satisfactorios. Por otro lado, la microabrasión del esmalte es un procedimiento bastante utilizado que remueve la capa superficial de la lesión con una pasta compuesta por ácido clorhídrico y piedra pómez. Con esta técnica puede ser erosionado un excesivo volumen de esmalte, aproximadamente hasta 360 $\pm 120 \mu \mathrm{m}$, por lo que no se considera esta técnica dentro del concepto de Odontología Mínimamente Invasiva (5).

Los infiltrantes resinosos son nuevos materiales disponibles en el mercado bajo el nombre comercial de ICON (DMG, Alemania), que fueron introducidos en la Odontología con el objetivo de paralizar la progresión de lesiones cariosas incipientes no cavitadas. Al aplicar los infiltrantes resinosos en estas lesiones, éstas pierden su apariencia blanquecina cuando las microporosidades han rellenado el cuerpo de la lesión, de esa forma dejándola con aspecto de esmalte sano (3).

El principio por el cual las lesiones de esmalte pueden ser enmascaradas con resinas infiltrantes se basa en el cambio de la dispersión de luz dentro de las lesiones. El esmalte sano tiene un índice de refracción (IR) de 1.62. Las microporosidades de las lesiones cariosas de esmalte pueden estar rellenas con agua (IR: 1.33) o con aire (IR: 1.0). La diferencia entre los índices de refracción de los cristales de hidroxiapatita del esmalte y el medio dentro de las microporosidades causan una dispersión de luz con apariencia opaca y blanquecina, especialmente cuando estas lesiones son desecadas. Las microporosidades infiltradas con resina presentan un IR de 1.46, y en contraste al medio acuoso, esta resina no puede evaporarse. La proximidad al IR de esmalte intacto sería el factor que hace que las lesiones infiltradas parezcan similares al tejido que las rodea (3).

La finalidad de la infiltración de las manchas blancas es el enmascaramiento estético no solamente inmediato, sino a largo plazo. Estudios han mostrado que las lesiones infiltradas podrían pigmentarse, debido a que el principal componente es el TEGDMA, un monómero con alta absorción de agua $y$, en consecuencia, de pigmentos. Sin embargo, la literatura científica relata que tal cambio de color puede ser resuelto realizando nuevamente el pulido de la lesión infiltrada (10).

Recientemente algunos autores han relatado diversas ventajas de la técnica de infiltración resinosa: estabilización mecánica del esmalte desmineralizado, sellado permanente de poros superficiales y áreas desmineralizadas profundas, preservación del tejido dental duro, y paralización del progreso de la lesión aumentando la resistencia a la desmineralización, riesgo mínimo de desarrollo de caries secundaria y alta aceptación de los pacientes (11). Se espera que la comercialización del infiltrante resinoso pueda expandirse a nivel global, pues no son todos los países que tienen acceso a este producto que por el momento es solamente desarrollado por la empresa alemana DMG bajo el nombre comercial de Icon.

\section{CONCLUSIÓN}

El uso de infiltrantes resinosos es eficaz para el tratamiento estético de las lesiones de manchas blancas leves ocasionadas por fluorosis. Esta técnica tiene como ventaja que es mínimamente invasiva ya que preserva el tejido dental a diferencia de otros procedimientos que desgastan la lesión de mancha blanca para obtener una mejora 
estética. En caso de un posible cambio de color o pigmentación se recomienda el pulido de las lesiones infiltradas. Es importante tener presente que el éxito estético del tratamiento de infiltración resinosa depende del grado de severidad de las lesiones, ya que para casos moderados o graves otros tratamientos demuestran mayor eficacia.

\section{REFERENCIAS}

1. Abanto Alvarez J., Rezende K. M., Marocho S. M., Alves F. B., Celiberti P., Ciamponi A. L. Dental fluorosis: exposure, prevention and management. Med Oral Patol Oral Cir Bucal. 2009; 14 (2): E103-7.

2. Patil M. M., Lakhkar B. B., Patil S. S. Curse of Fluorosis. Indian J Pediatr. 2018; 85 (5): 375-83.

3. Paris S., Meyer-Lueckel H. Masking of labial enamel white spot lesions by resin infiltration-a clinical report. Quintessence Int. 2009; 40 (9): 713-8.

4. Ardu S., Castioni N. V., Benbachir N., Krejci I. Minimally invasive treatment of white spot enamel lesions. Quintessence Int. 2007; 38(8): 633-6.

5. Tong L. S., Pang M. K., Mok N. Y., King N. M., Wei S. H. The effects of etching, micro- abrasion, and bleaching on surface enamel. J Dent Res. 1993; 72 (1): 67-71.

6. Paris S., Meyer-Lueckel H., Cölfen H., Kielbassa A. M. Resin infiltration of artificial enamel caries lesions with experimental light curing resins. Dent Mater J. 2007; 26 (4): 582-8.

7. Paris S., Meyer-Lueckel H., Cölfen H., Kielbassa A. M. Penetration coefficients of commercially available and experimental composites intended to infiltrate enamel carious lesions. Dent Mater. 2007; 23 (6): 742-8.

8. Neuhaus K. W., Graf M., Lussi A., Katsaros C. Late infiltration of post-orthodontic white spot lesions. J. Orofac Orthop. 2010; 71 (6): 442-7.

9. FDI Policy Statement. Minimal Intervention in the Management of Dental Caries. FDI General Assembly: October 2002. Vienna.

10. Borges A., Caneppele T., Luz M., Pucci C., Torres C. Color stability of resin used for caries infiltration after exposure to different staining solutions. Oper Dent. 2014; 39 (4): 433-40.

11. Paris S., Lausch J., Selje T., Dörfer C. E., Meyer-Lueckel H. Comparison of sealant and infiltrant penetration into pit and fissure caries lesions in vitro. J Dent. 2014; 42 (4): 432-8. 